\title{
Multiplication of a Multichannel Notch Filter Based on a Phase-Shifted Phase-Only Sampled Fiber Bragg Grating
}

\author{
Ming Li, Student Member, IEEE, Takeo Fujii, and Hongpu Li, Member, IEEE
}

\begin{abstract}
By utilizing the multiple phase-shift and the sampling techniques, we demonstrate, for the first time, a novel multichannel notch filter with a multipliable channel count. Three kinds of notch filters with channel counts of 51, 102, and 153 are obtained by thermally inducing one, two, and three $\pi$ phase-shifts into a 51-channel phase-only sampled fiber Bragg grating, respectively. The proposed technique provides the potential applications to the multiwavelength fiber laser, the multichannel all-optical logic devices, and an optical code-division multiple access system.
\end{abstract}

Index Terms-Multichannel notch filter, multiplication, phaseonly sampled fiber Bragg grating (FBG), phase shift.

\section{INTRODUCTION}

A TTRIBUTED to the unique advantages such as the small size, low loss, high reliability, and compatibility with the fiber-type components, fiber Bragg gratings (FBGs) have attracted great interest for the applications to optical communications, sensing, and microwave photonic systems, etc. [1]-[14]. With the development of all-optical switching and the wavelength-division-multiplexing technique, the sampled FBGs and multiple-phase-shift FBGs have been utilized as either the multichannel filter for the dispersion compensator [5]-[9], or the optical logic devices [10], [11]. The multiple phase-shifted FBG exhibits unique advantage for the generation of multiple ultranarrow response in transmission spectrum. In particular, with suitably multiple phase-shifts inserted, various kinds of spectra such as a quasi-flat-top transmission spectrum have been obtained [12]. Moreover, since the phase shifts in a grating can be easily introduced by simply using either the thermal or the mechanical approach, which makes the phase shifted FBG extremely flexible [11], [14]. However, the FBG with both a high channel count and high channel uniformity is hardly obtained with the above methods. To solve the issue, the sampled FBGs, including the phase-only, the amplitude-only, or the simultaneous phase- and amplitude-sampling, have been proposed and

Manuscript received January 08, 2009; revised March 24, 2009. First published April 21, 2009; current version published June 17, 2009. This work was supported in part by a Grant-in-Aid for Scientific Research from the Japan Society for the Promotion of Science, in part by the Kurata Memorial Hitachi Science and Technology Foundation, in part by the International Communications Foundation, and in part by Hamamatsu Science and Technology Promotion in Japan.

The authors are with the Department of Electrical and Electronic Engineering, Shizuoka University, Hamamatsu 432-8561, Japan (e-mail: dhli@ipc.shizuoka.ac.jp).

Color versions of one or more of the figures in this letter are available online at http://ieeexplore.ieee.org.

Digital Object Identifier 10.1109/LPT.2009.2020684

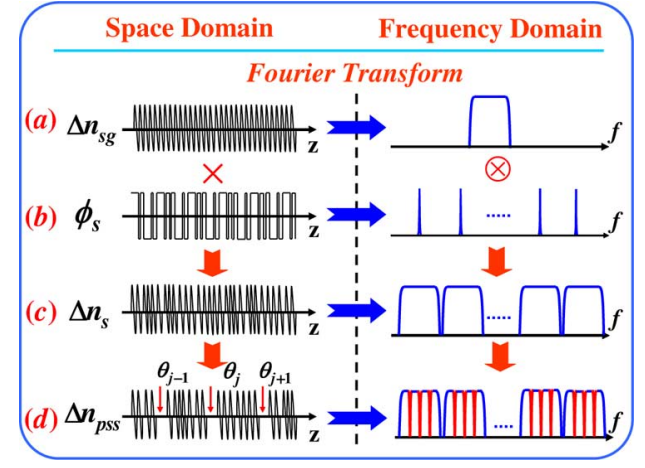

Fig. 1. Fourier analysis of the channel count multipliable multichannel notch filter based on multiple-phase-shifted phase-only sampled FBG.

widely used, which enable one to systematically design any kind of FBG with both the ideal channel performances and a high channel count [5]-[9]. Most recently, we have proposed a technique for the realization of a multichannel notch filter by using a thermally induced phase-shifted phase-only sampled FBG. However, only one phase-shift is introduced into the phase-only sampled FBG [14]. In this letter, by introducing multiple phase-shifts into the sampled FBG, we demonstrate, for the first time to our knowledge, a novel multichannel notch filter with a multipliable channel count.

\section{PRINCIPLE AND EXPERIMENTAL SETUP}

Fig. 1 illustrates the Fourier analysis for the multiplication of the multichannel notch filter by utilizing a multiple-phaseshifted phase-only sampled FBG. As is generally known, the phase-only sampled FBG is the product of a single-channel seed grating $\Delta n_{\mathrm{sg}}$ [as shown in Fig. 1(a)] with a phase-only sampling function $s(z)=\exp \left(i \phi_{s}(z)\right)$ [as shown in Fig. 1(b)]. The induced refractive index-modulation $\Delta n_{s}$, as is shown in Fig. 1(c), can be expressed as [9]

$$
\begin{aligned}
\Delta n_{s}(z)=\operatorname{Re}\left\{\frac{\Delta n_{1}(z)}{2} \exp (\right. & i \frac{2 \pi z}{\Lambda} \\
& \left.\left.+i \phi_{g}(z)+i \phi_{s}(z)\right)\right\} .
\end{aligned}
$$

Here $\Delta n_{1}(z)$ is the maximum index-modulation, $z$ is the position along the grating, $\Lambda$ represents the average uniform period of the seeding grating, and $\phi_{g}(z)$ represents the phase-change of the seed grating related to the grating chirp. $\phi_{S}(z)$ denotes 


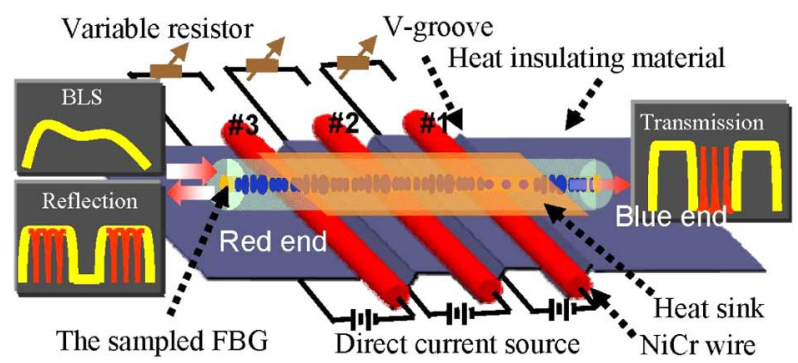

Fig. 2. Schematic diagram of the proposed channel count multipliable multichannel notch filter. BLS: broadband light source.

the phase profile of the phase-only sampling function. For convenience, the "dc" part of the index-modulation is neglected in (1).

Once the phase shifts $\theta_{j}(j=1,2, \ldots, N)$ are introduced into a phase-only sampled linearly chirped FBG at the positions of $z_{j}$, the index-modulation $\Delta n_{\text {pss }}$ [as shown in Fig. 1(d)] can be expressed as

$$
\begin{array}{r}
\Delta n_{\mathrm{pss}}(z)=\operatorname{Re}\left\{\frac { \Delta n _ { 1 } ( z ) } { 2 } \operatorname { e x p } \left(i \frac{2 \pi z}{\Lambda}+i \phi_{g}(z)+i \phi_{s}(z)\right.\right. \\
\left.\left.+i \sum_{j}^{N} \theta_{j} \cdot q\left(z-z_{j}\right)\right)\right\}
\end{array}
$$

where $N$ is the number of the phase shifts and the function $q$ is defined as: $q\left(z-z_{j}\right)=0$ if $z<z_{j}$ and $q\left(z-z_{j}\right)=1$ if $z \geq z_{j}$. It can be seen from (2) that the multiple phase-shifted phase-only sampled FBG is equivalent to a multiple phase-shift seed grating multiplied by a sampling function in the spatial domain. Therefore, the reflection spectrum resulted from a multiple phase-shifted seed grating should be copied simultaneously through all the resulted channels. As is shown in Fig. 1(d), particular three-notch responses in each channel resulted from the sampled FBG may be realized by introducing three $\pi$ phase shifts. As a result, the channel count of the resulted notch filter is multiplied by the number of phase-shifts in a linearly chirped sampled FBG.

To confirm the above proposal, an experiment to thermally induce multiple phase-shifts into a phase-only sampled FBG is introduced as follows. Fig. 2 shows the schematic diagram of our setup. To realize multiplication of the channel count, more than one $\mathrm{NiCr}$ wire heater is utilized and fixed in the $\mathrm{V}$-grooves in order to produce multiple phase-shifts based on the thermal-optical and thermal expansion effect. In particular, three $\mathrm{NiCr}$ wires labelled as \#1, \#2, and \#3 are employed in this case. Here the phase-only sampled 51-channel FBG is a linearly chirped grating designed and fabricated to be used as the simultaneous dispersion and dispersion-slope compensator, which has a channel spacing of $0.8 \mathrm{~nm}$ and a grating length of $12 \mathrm{~cm}$ [8], [13]. Note that the reason for using such a linearly chirped FBG is that the wavelengths with an identical detuning from the center of each channel are reflected at almost the same position of the grating; i.e., the notch wavelengths resulted have
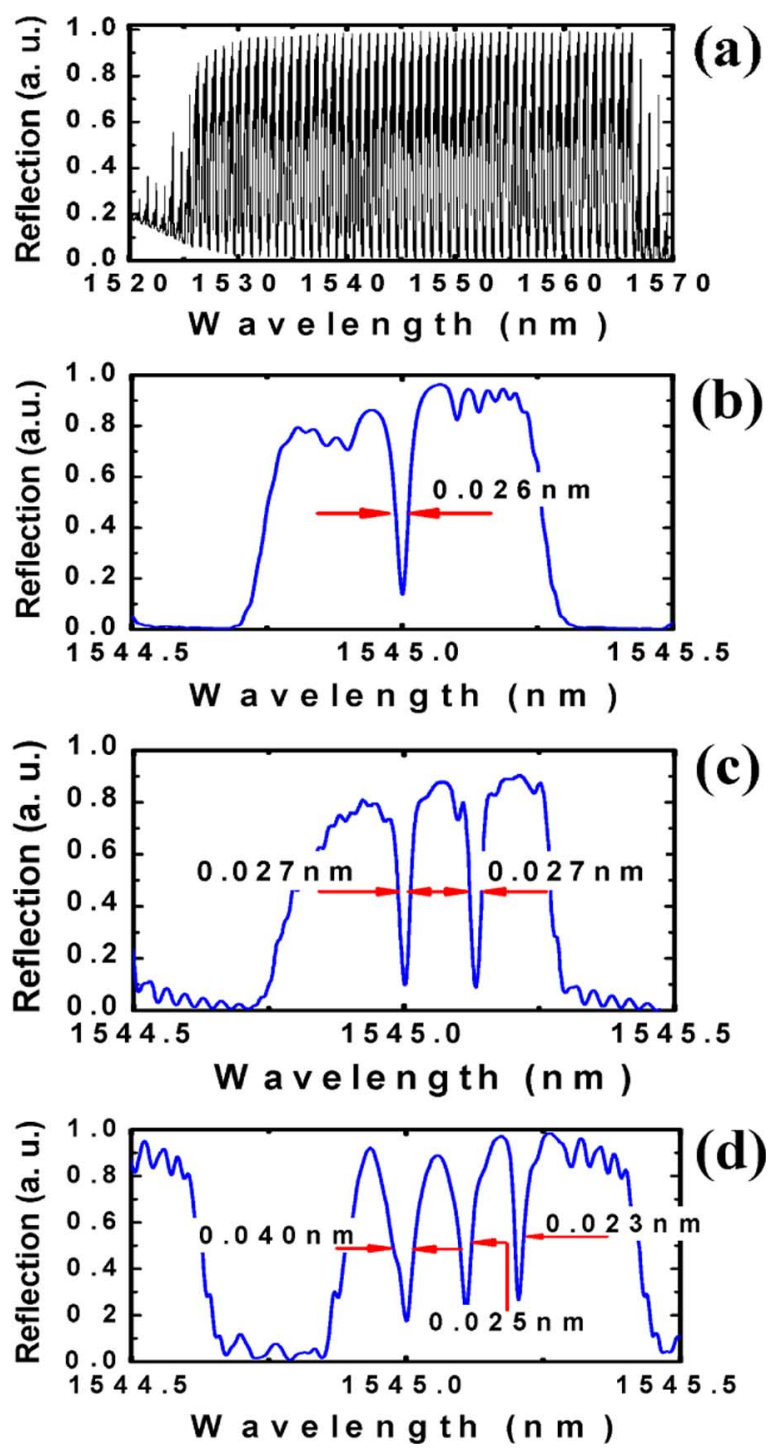

Fig. 3. Measurement results for the $\pi$ phase-shifted 51-channel FBG. (a) 51-channel reflection spectrum with three $\pi$ phase-shifts, (b) the central channel reflection spectrum with one $\pi$ phase-shift, (c) the central channel reflection spectrum with two $\pi$ phase-shifts, and (d) the central channel reflection spectrum of Fig. 3(a).

a linear relationship with the grating position, which in turn means that notch filters in all the channels are simultaneously obtained even if only one phase-shift is inserted in the FBG. In the experiment, the left and right sides of the FBG are related to the long and short wavelength ends, respectively. By tuning the variable resistors, temperature change within each local small section of fiber may be introduced at that point which means that the magnitude of the phase-shift could be continuously tunable. Here an amplified spontaneous emission is used as the broadband light source. A heat sink made from a thin copper plank is placed over the crossing area to dissipate the unwanted heat which partly determines the stability of the notch filter.

\section{RESUlTS AND DISCUSSION}

Fig. 3 shows the measurement results for a linearly chirped 51-channel FBG with three different numbers of $\pi$ phase-shifts. Fig. 3(b) shows the reflection spectrum as only one $\pi$ phase-shift 


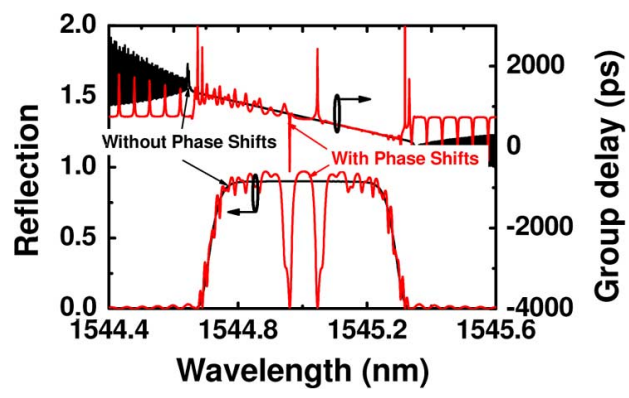

Fig. 4. Simulation results of the reflection and group delay spectra with and without two $\pi$ phase-shifts.

is inserted; it can be seen that the notch filter with a narrow bandwidth of $0.026 \mathrm{~nm}$ is obtained. The $\pi$ phase-shift in Fig. 3(b) is implemented only by turning on the \#3 wire, i.e., the closest one to the red end of the FBG. Fig. 3(c) and (d) shows the results where \#1-\#2 and \#1-\#3 wires are heated up, respectively. Fig. 3(a) and (d) illustrates the reflection spectra of the 51-channel FBG and the central channel under the condition of three $\pi$ phase-shifts being inserted. It can be seen that three narrow notch filters with different bandwidths of 0.040, 0.025, and $0.023 \mathrm{~nm}$ are obtained. Compared with Fig. 3(b), it is obviously seen that the channel count of the notch filter in each FBG channel is linearly proportional to the number of the phase-shifts inserted. Therefore, three kinds of multichannel notch filters with the channel counts of 51,102, and 153 have been achieved just by thermally inducing one, two, and three $\pi$ phase-shifts into a 51-channel phase-only sampled FBG, respectively. Note that, although the extinction ratios shown in the experimental results are smaller than the theoretical ones, it is high enough for some practical applications such as the multiwavelength fiber laser [15].

In addition to the above results, three points should be noted here. First, unlike the one-phase-shifted FBG, there exist small oscillations on the spectra which can be clearly seen at the bottom line of Fig. 3(c) and (d). These oscillations are enhanced with increment of the phase shifts, which is caused by the multiple reflections between each $\pi$ phase-shift (i.e., a Fabry-Pérot cavity is formed between each paired $\pi$ phase-shifts). Second, from the Fig. 4 which shows the simulation results of the reflection and group delay spectra with and without two $\pi$ phase-shifts, it is found that once the two phase-shifts are introduced, two peaks on the group delay spectrum appear just at the notch wavelengths. Finally, as shown in Fig. 3(d), one can find that the bandwidth of the reflection spectrum of the phase-only sampled FBG is obviously increased. However, in the simulation results, this phenomenon cannot be seen. Therefore, we believe that this broadening is mainly caused by the existence of the thermal expansion effect in the fiber. In our experiment, since only one heat-sink is utilized, the heat resulted from the three $\mathrm{NiCr}$ wires cannot be uniformly and rapidly dissipated compared with that utilizing a single wire heater, and thus the fiber temperature is largely increased, which in return means that the scheme utilizing more than three wires in our case may be insufficient for the practical application by using the present heat-diffusion approach. Further studies to improve the heat dissipation efficiency and the temperature stability of the thermally induced multiple phase-shifts are currently under way.

\section{CONCLUSION}

A novel multichannel notch filter with a multipliable channel count is theoretically and experimentally demonstrated by utilizing both multiple-phase-shift and sampling techniques. Three multichannel notch filters with channel counts of 51,102, and 153 are obtained by thermally inducing one, two, and three $\pi$ phase-shifts into a 51-channel linearly chirped FBG, respectively. The proposed technique may find the potential applications to the multiwavelength fiber laser, multichannel all-optical logic devices, and the optical code-division multiple access system.

\section{REFERENCES}

[1] F. Ouellette, P. A. Krug, T. Stephens, G. Dhosi, and B. Eggleton, "Broadband and WDM dispersion compensation using chirped sampled fiber Bragg gratings," Electron. Lett., vol. 31, pp. 899-901, May 1995.

[2] Y. J. Rao, "In-fibre Bragg grating sensors," Meas. Sci. Technol., vol. 8, pp. 1442-1462, Apr. 1997.

[3] F. Zeng and J. Yao, "Ultrawideband impulse radio signal generation using a high-speed electrooptic phase modulator and a fiber-Bragggrating-based frequency discriminator," IEEE Photon. Technol. Lett., vol. 18, no. 19, pp. 2062-2064, Oct. 1, 2006.

[4] Melloni, M. Chinello, and M. Martinelli, "All-optical switching in phase-shifted fiber Bragg grating," IEEE Photon. Technol. Lett., vol. 12 , no. 1 , pp. $42-44$, Jan. 2000.

[5] M. Ibsen, M. K. Durkin, M. J. Cole, and R. I. Laming, "Sinc-sampled fiber Bragg gratings for identical multiple wavelength operation," IEEE Photon. Technol. Lett., vol. 10, no. 6, pp. 842-844, Jun. 1998.

[6] V. Buryak, K. Y. Kolossovski, and D. Y. Stepanov, "Optimization of refractive index sampling for multichannel fiber Bragg gratings," IEEE J. Quantum Electron., vol. 39, no. 1, pp. 91-98, Jan. 2003.

[7] H. Li, Y. Sheng, Y. Li, and J. E. Rothenberg, "Phase-only sampled fiber Bragg gratings for high-channel-count chromatic dispersion compensation," J. Lightw. Technol., vol. 21, no. 9, pp. 2074-2083, Sep. 2003.

[8] M. Morin, M. Poulin, A. Mailloux, F. Trépanier, and Y. Painchaud, "Full $C$-band slope-matched dispersion compensation based on a phase sampled Bragg grating," in Proc. OFC 2004, Los Angeles, CA, 2004, Paper WK1.

[9] H. Li, M. Li, Y. Sheng, and J. E. Rothenberg, "Advances in the design and fabrication of high channel-count fiber Bragg gratings," J. Lightw. Technol., vol. 25, no. 9, pp. 2739-2749, Sep. 2007.

[10] N. K. Berger, B. Levit, B. Fischer, M. Kulishov, D. V. Plant, and J. Azaña, "All-optical temporal differentiator based on a single phaseshifted fiber Bragg grating," Opt. Express, vol. 15, pp. 371-381, Jan. 2007.

[11] S. Y. Li, N. Q. Ngo, S. C. Tjin, P. Shum, and J. Zhang, "Thermally tunable narrow-band-pass filter based on a linearly chirped fiber Bragg grating," Opt. Lett., vol. 29, pp. 29-31, Jan. 2004.

[12] L. Wei and J. W. Y. Lit, "Phase-shifted Bragg grating filters with symmetrical structures," J. Lightw. Technol., vol. 15, no. 8, pp. 1405-1410, Aug. 1997.

[13] M. Li and H. Li, "Reflection equalization of the simultaneous dispersion and dispersion-slope compensator based on a phase-only sampled fiber Bragg grating," Opt. Express, vol. 16, pp. 9821-9828, May 2008.

[14] M. Li, H. Li, and Y. Painchaud, "Multi-channel notch filter based on a phase-shift phase-only sampled fiber Bragg grating," Opt. Express, vol. 16, pp. 19388-19394, Nov. 2008.

[15] M. Li, H. Li, and Y. Painchaud, "Tunable high channel-count notch filter based on a phase-shift phase-only sampled FBG and its application to multi-wavelength fiber laser," in Proc. OFC 2009, San Diego, CA, 2009, Paper OWT5. 